\title{
Recognition of Interactions with Objects based on Radio Modules
}

\author{
Axel Czabke, Jakob Neuhäuser, Tim C. Lueth \\ Department of Micro Technology and Medical Device Technology \\ Technical University Munich \\ Munich, Germany \\ axel.czabke@tum.de
}

\begin{abstract}
In this contribution a novel system for the recognition of interaction with objects is presented. The developed system iActionlogger (interActionlogger) consists of radio modules that are used to recognize contact situations between a user and an object and is meant as a monitoring tool for users activities. Worldwide a great effort is done in developing technologies that allow elderly people to live independent and yet secure in their home environment for as long as possible. The recognition of Activities of Daily Living (ADLs) is an important point in order to evaluate the overall health status of a person. At this time no technology could win through to be the tool to reliably work in a realistic environment. In this paper we compare some of the existing technologies and evaluate our approach in respect to technical and user demands.
\end{abstract} care

Keywords-interaction with objects; recognition of ADL; elderly

\section{INTRODUCTION}

Most developed countries will face the same set of healthcare challenges in the upcoming years. The demographical, structural and social trends tend towards an increasing number of elderly people whereas the number of the young and working people is decreasing. This will create dramatic impact on society affecting both financial and organizational aspects [1].

From the medical point of view there are different reasons for becoming a nursing case. Physical limitations are widely prevalent in aging individuals and easiest to detect. A reduction in physical efficiency is a consequence of the normal aging process and does not necessarily have to lead to severe problems. Early detection and support of physical limitations are most often helpful, in order to keep everyday complications in check. Vegetative limitations refer to the degradation of the body's own self-regulatory system of vital functions. These can emerge separate from physical or mental disruptions. The most common are illnesses of the cardio-vascular system. By means of early recognition and corresponding adjustment in one's lifestyle, severe consequences can, for the most part, be decidedly protracted, or even completely avoided. Mental efficiency also decreases with age. Even individuals who are in perfect physical shape can develop cognitive deficits. Early support for such individuals can assure that daily life within their familiar environment can continue to be possible, thereby avoiding an abatement of cognitive and physical capacities. Dementia plays a prominent role in the field of mental or cognitive deficits. It is a progressive, disabling, chronic disease affecting $5 \%$ of all persons above 65 and over $40 \%$ of people over 90 years [2]. Today we know that the probability of suffering from dementia grows with the age of a person. We also know that the average life expectancy is increasing. As consequence of these facts and the demographical development a massive gain of dementia is to be expected during the next decades. Due to the reduced ratio of carers vs. nursing cases new strategies have to be developed. This arouses the need for new technologies that enable elderly people to live their life as long as possible in their own places, which would lead to the two following benefits. First it would relieve nurses, carers and with the related saving of costs the health system. Second it would help people to spend their evening of life in their familiar surroundings, which most elderly people aspire. [3] suggested that most of them prefer staying at home for as long as possible, even if they are at risk. To identify possible risks or first changes in behaviour the monitoring of every day activities is the most common way. For that purpose, two specific sets of activities have been defined [4]. One describes the Activities of Daily Living (ADL) and focuses on the functional status of a person. The other one, the Instrumental Activities of Daily Living (IADL), describes interaction with physical and social environment. An automated recognizing of ADLs would provide an objective tool to assess self maintenance abilities and to make slight changes visible and thus help in the early diagnoses of illness. We propose an approach that allows the detection of interacting with objects or persons and thus is capable to document the execution and the incidence of some vital ADLs such as toileting or getting a drink. The idea is to interpret interaction with objects as the corresponding action. For example, "drinking coffee" can be inferred from a person's interaction with "coffee machine" and "mug". The precondition for this approach is to have a tool or system that is capable of recognizing interaction in a reliable way without being noticed by the user as bothersome.

\section{STATE OF THE ART}

The detection of interaction with objects is not a new idea. Thus there are various approaches that try to address the challenges involved with a robust automated monitoring of contact situations between persons and objects or objects and objects. The field of application reaches from autonomous robots to medical uses and the detection of ADLs. To provide an overview we clustered the existing approaches in respect of 
the technology used. This leads to a subdivision of detection of interaction as shown in figure 1. The sub items are infrared, ultrasonic sound, visual systems and radio systems.

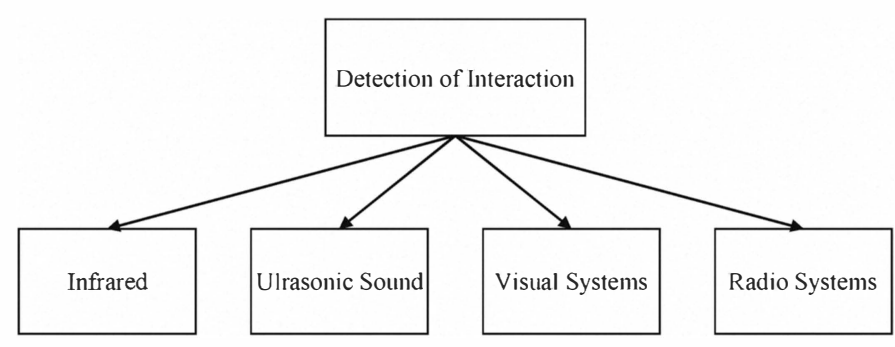

Figure 1. Subdivision of Detection of Interaction.

In 1995 [5] and [6] developed the system LOCISS (Locally Communicable Infrared Sensory System) for interaction between mobile robots. To avoid collision between robots they had to be able to recognize each other. As the use of radio communication with no possibility to reduce the transmission range led to a massive signal disturbance, they developed a local communication system based on infrared. Every robot is sending its own ID via infrared and has an infrared sensory system to receive and interpret infrared signals. If the signal is reflected by an object, the robot will receive its own ID and thus interpret the object as a non moving obstacle and change its direction to drive around it. If two robots meet they will recognize the ID of each other and start exchanging information about their planed route to avoid collision. The original transmitting distance of the infrared signal was about 6 metres. It turned out to be of a big advantage to reduce the transmission distance to 1 meter. With this configuration only obstacles and robots close enough to be of relevance were detected. Besides systems that are used for robots, systems that can locate people or objects in buildings are of special interest. One of these approaches is based upon the use of infrared beacons. Because of the low costs and the high availability of infrared applications they are used in many projects. One of the first publications in this area is a system called Active Badge by [7] and [8]. They developed a small device $(55 \mathrm{~mm} \times 55 \mathrm{~mm}$ $x 7 \mathrm{~mm}$ ) that transmits a 0,1 seconds lasting infrared signal with a range of 6 metres every 15 seconds. The signals are received by a sensory network and sent to a processing unit. As every Active Badge sends out a unique infrared signal, the processing unit can distinguish between different Active Badges and their position. An advantage of the system is that due to the short transmission period the energy consumption is very low which enables a long battery life time (up to one year). Furthermore the probability of interfering signals is very little. With regard to an every day use by people at home the fact that the system needs visual contact between transmitter and receiver must be evaluated as disadvantage as this would mean that people would always have to wear the badge on top of their clothes. An infrared system based on PIRs (Presence Infrared Sensors) is presented by [9]. They prepared two smart homes with PIRs, which were positioned in a way that they could oversee certain areas of interest, e.g. bed, armchair, entrance, sink and cabinet. The sensors fire whenever they detect movement in their sector. Every sensor is assigned to a certain activity. For example, a firing of the sensor covering the bed is interpreted as "sleeping" whereas a firing of the sensor covering the sink is interpreted as "washing". The data of all sensors is transmitted to a computer that saves and analyzes the information. The PIRs are not expensive and easy to install but they cannot distinguish between different users or humans and pets. In [10] a system based on ultrasonic sound is presented. Here the participants of a study were wearing small devices called Active Bats transmitting ultrasonic sound. Ultrasonic sound receivers are integrated in the ceiling of the test environment and connected to a sensor network. If a request is sent by the basis computer, the requested Active Bat answers and its position can be calculated from the runtime of the ultrasonic signal to the different sensors in the ceiling. An inverted functional principle is presented in [11]. Here the mobile device is receiving ultrasonic signals from fixed active beacons to calculate the actual position. This enables the use of many listeners at the same time. Both systems ([10] and [11]) are prone to problems caused by unwanted reflections of the ultrasonic sound and demand the user to wear the mobile device on top of his/her clothing. A system based on the use of computer vision techniques to augment home based sensorized environments was introduced by [12]. They equipped a smart home environment kitchen with a video camera. The obtained video information was processed by an algorithm to distinguish between different persons and their actions in the kitchen. As a result they stated that video analysis alone is not sufficient due to the lack of information associated with occlusions. Nevertheless it can significantly support data from other sensors by attaching the identity to persons performing the task and interacting with the sensor. Another approach based on video technologies is presented in [13]. Here the authors propose a Ubiquitous Camera Interface Framework (UCIF) to address issues dealing with distributed visual processing for a personal service robot in an intelligent home environment. Radio Frequency Identification (RFID) tags and readers are used in several activity recognition systems because of their durability, small size and low costs. You can distinguish between systems that are based on fixed readers and tagged moving objects as shown in [14] and systems with mobile readers worn by the user. The iGlove and the iBracelet that are presented in [15] are examples for the latter ones. [16] also integrated an RFID reader into a glove to position the antenna as close as possible to the tags of grasped objects. The systems based on mobile readers have a very limited read range with a maximum of 10 centimetres. Despite the great progress in RFID technology during the last few years, the physical restrictions for the reading range are inevitable. Especially for mobile RFID readers it is very important to find a good balance between antenna size, power consumption and reading frequency to name only a few of the relevant parameters.

As the examples mentioned above show, various approaches to the interaction recognition problem can be found in literature. Some of them, e.g. the PIR system, use positioning to infer activity or interaction with objects. Others like RFID are not bound to fix positions. The examples are to represent different technical strategies, which can and already have been combined with each other and/or smart home environments. Each of the technologies has its own advantages and disadvantages that are summarized in Table 1. 


\begin{tabular}{|l|l|l|}
\hline Technology & \multicolumn{1}{|c|}{ Advantage } & \multicolumn{1}{c|}{ Disadvantage } \\
\hline Infrared & $\begin{array}{l}\text { low costs } \\
\text { low energy consumption }\end{array}$ & $\begin{array}{l}\text { System must be worn on top of } \\
\text { clothing } \\
\text { PIR: No distincion between } \\
\text { different persons }\end{array}$ \\
\hline $\begin{array}{l}\text { Ultrasonic } \\
\text { Sound }\end{array}$ & $\begin{array}{l}\text { high accuracy in } \\
\text { positioning }\end{array}$ & $\begin{array}{l}\text { System must be worn on top of } \\
\text { clothing } \\
\text { Reflection of Objects }\end{array}$ \\
\hline $\begin{array}{l}\text { Visual } \\
\text { Systems }\end{array}$ & $\begin{array}{l}\text { user is not affected by } \\
\text { any device } \\
\text { occlusions } \\
\text { quality depends on light settings } \\
\text { intensive processing of data }\end{array}$ \\
\hline RFID & $\begin{array}{l}\text { low cost } \\
\text { easy tagging of objects }\end{array}$ & $\begin{array}{l}\text { limited reading range } \\
\text { User must wear glove }\end{array}$ \\
\hline
\end{tabular}

TABLE I. ADVANTAGES AND DisADVANTAGES OF DisCUSSED TECHNOLOGIES

\section{TASK AND NEW APPROACH}

The task to develop a system for robust measuring of interactions used by elderly people contains several demands that should be met. Probably the most important one is the ease of use. Especially elderly people are used to do certain things in their accustomed way. They will hardly accept and use systems that only work if they change their behaviour according to the demands of the system. Thus the modus operandi must be to create sensors and devices that do not dictate a certain carrying out of ADLs. Yet the systems have to be robust, because users can perform activities in various ways. Devices, models of activities and recognition software should be designed in respect of that variety. Even though people differ in performing activities, most agree in their preference for systems that are working imperceptible in the background. There are two comprehensible reasons for that. First people do not want to get disturbed in their usual way of living by a complicated device that reminds them of their own need for help all the time. Second when interacting with other people they don't want to be stigmatized as care needing because of a peculiar assisting device. That leads to the demand of small devices that are not attracting attention. In the ideal case devices are not recognized as such even by the user. Yet they have to be reliable, easy to install in different environments and of limited maintenance requirement. Of course, from the future user's point of view the costs also play a prominent role in the decision process for or against the use of a technical assistance system. With respect to this point technologies that are suitable to be manufactured as a mass product seem more promising to develop affordable devices for "real users" than expensive proprietary high tech solutions.

We propose an approach that addresses these demands and proved to be promising in the detection of interaction with objects and hence in the automatic detection of some ADLs. Our key technology are radio modules that recognize "contact" between each other. The range of the radio modules can be set by software to a value from 0,3 metres -40 metres. Hence the interaction radius $r_{I}$ can be defined for different setups individually. This is a vital point in order to develop a system that does not affect the user when executing his/her activities. It opens up the possibility to provide the user with a device that unlike an RFID glove can be worn understated in a pocket an yet has the power to detect interaction with marked objects.
Every module sends out an identification number (ID) with a set frequency $\mathrm{f}_{\mathrm{T}}$. The duration for transmitting the ID $\mathrm{d}_{\mathrm{T}}$ is 1 ms. The receiving frequency $f_{R}$ and the receiving duration $d_{R}$ must be chosen according to $f_{T}$ and $d_{T}$. We defined two types of devices: active motes (AM) and passive motes (PM). The names "active" and "passive" refer to the ability to "listen" to other motes. Passive motes only send their ID with $\mathrm{f}_{\mathrm{T}}$ and do not switch to receiving mode. In contrast to this the active motes switch with $f_{R}$ to receiving mode and save every contact event $\varepsilon_{\mathrm{I}}$ within $\mathrm{d}_{\mathrm{T}}$ to a flash memory. $\varepsilon_{\mathrm{I}}$ contains the ID of the detected mote $m_{x}$ and a unix timestamp.

$$
\varepsilon_{\mathrm{I}} \in\left\{\mathrm{m}_{\mathrm{x}}, \mathrm{t}\right\}
$$

Figure 2 shows an example of two motes $m_{1}$ and $m_{2}$ in a varying distance. Depending on the affiliation of $m_{1}$ and $m_{2}$ the scenario can be described as following:

- Case 1: One active mote and one passive mote:

$$
\begin{gathered}
\mathrm{m}_{1} \in \mathrm{AM}, \mathrm{m}_{2} \in \mathrm{PM} \\
\operatorname{Distance}\left(\mathrm{m}_{1}, \mathrm{~m}_{2}\right)>\mathrm{r}_{\mathrm{I} 1}, \mathrm{r}_{\mathrm{I} 2} \Rightarrow \varepsilon_{1}=\{\} \\
\mathrm{r}_{\mathrm{I} 1}>\operatorname{Distance}\left(\mathrm{m}_{1}, \mathrm{~m}_{2}\right)>\mathrm{r}_{\mathrm{I} 2} \Rightarrow \varepsilon_{1}=\{\} \\
\operatorname{Distance}\left(\mathrm{m}_{1}, \mathrm{~m}_{2}\right)<\mathrm{r}_{\mathrm{I} 1}, \mathrm{r}_{\mathrm{I} 2} \Rightarrow \varepsilon_{1}=\left\{\mathrm{m}_{2}, \mathrm{t}\right\}
\end{gathered}
$$

- Case 2: Two active motes:

$$
\begin{gathered}
\mathrm{m}_{1}, \mathrm{~m}_{2} \in \mathrm{AM} \\
\operatorname{Distance}\left(\mathrm{m}_{1}, \mathrm{~m}_{2}\right)>\mathrm{r}_{11}, \mathrm{r}_{\mathrm{I} 2} \Rightarrow \varepsilon_{1}=\{\}, \varepsilon_{2}=\{\} \\
\mathrm{r}_{\mathrm{I} 1}>\operatorname{Distance}\left(\mathrm{m}_{1}, \mathrm{~m}_{2}\right)>\mathrm{r}_{\mathrm{I} 2} \Rightarrow \varepsilon_{1}=\{\}, \varepsilon_{2}=\left\{\mathrm{m}_{1}, \mathrm{t}\right\} \\
\operatorname{Distance}\left(\mathrm{m}_{1}, \mathrm{~m}_{2}\right)<\mathrm{r}_{\mathrm{I} 1}, \mathrm{r}_{\mathrm{I} 2} \Rightarrow \varepsilon_{1}=\left\{\mathrm{m}_{2}, \mathrm{t}\right\}, \varepsilon_{2}=\left\{\mathrm{m}_{1}, \mathrm{t}\right\}
\end{gathered}
$$
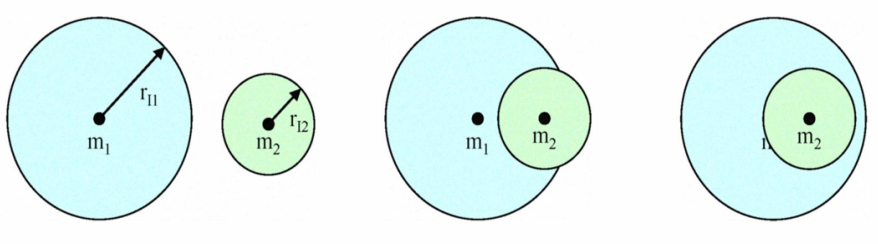

Figure 2. Two motes with different interaction radii $r_{I}$ in varying distances.

As the transmission range (interaction radius) and affiliation of a mote (passive or active) are defined by software, the system can be seen as a very flexible construction kit. Motes can be attached to different objects and according to the demands of the surroundings their range can be set. A vital advantage of the system compared to RFID is that beside the possibility to adopt the reading range as needed, the conventional classification into marker (tag) and reader gets expanded. Whereas the passive mote corresponds to a marker, or to say it in RFID terms, a "tag", the active mote combines both functions reader and marker in one device. This opens up 
the possibilty to create intelligent objects that are recognized by others and also memorize whom they had contact with. To assign this to the recognition of ADLs, it opens up the possibility to also monitor the frequency of social contacts which is a very good indicator for one's physical activity and constitution.

\section{SYSTEM ARCHITECTURE}

\section{A. Normal Operation}

The system contains of four different device types that will be described in the following. In normal operation mode as shown in figure 3, active and passive motes transmit their ID with $f_{T}$ within the individually set range. The active motes recognize contact to other active or passive motes. The corresponding ID and time information is saved by the active mote. To read out memory the active mote must be connected to a computer. This can either be done by wire or wireless. For the latter way we developed a basis mote that automatically triggers wireless data transmission whenever an active motes is recognized in a range of less than 0,3 metres. The computer is used to visualize and analyze the recorded data.

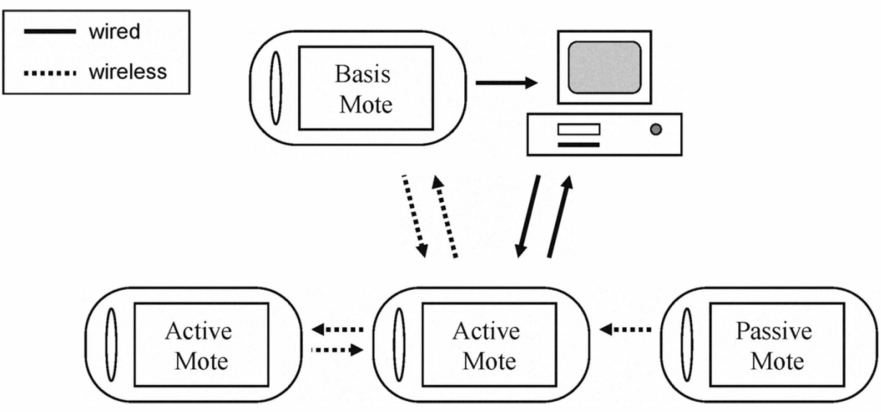

Figure 3. System architecture in normal operation mode.

\section{B. Initializing}

As already mentioned, an important feature of our interaction recognition system is the setting of the transmission range of passive motes und thus the definition of the interaction radius. This is done in the initializing mode. The idea is that the installation of the system is easy and can be done without the use of PC. Due to energy consumption and their small size the motes do not have buttons or a display as user interface. That is why an extra initializing device was developed. Its key function is to offer an easy user interface for a quick setting of the transmission range. When installing the system, the initializing device first searches for active motes in the environment. All found active motes are shown on the display and the user selects one of them. In the next step the chosen active mote searches for passive motes and sends the IDs of all found passive motes to the initializing device. Now the user selects the desired passive mote and the transmission range of the passive mote is automatically adapted to the distance between the selected motes and saved to the EEPROM of the passive mote. The distance is defined as the new interaction radius $r_{I}$ of the passive mote (Figure 4.)

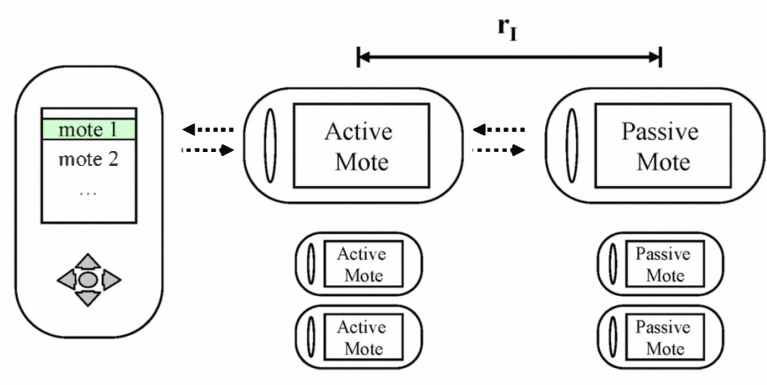

Figure 4. Setting interaction radius $r_{I}$ with the initializing device.

\section{Hardware}

The system consists of four different devices:

- Active motes,

- Passive motes,

- A basis mote,

- And the initializing device.

To achieve a maximum of flexibility, all of them are based on the same hardware platform shown in Figure 5. The principal component is the radio module NanoLOC AVR from Nanotron. It consists of a $2,4 \mathrm{GHz}$ radio chip (1) and a microcontroller (2). The module is connected to a triaxial acceleration sensor (3) to detect motion and a flash-EEPROM (4) to save data. The real-time-clock (RTC) (5) provides the microcontroller with time information via $\mathrm{I}^{2} \mathrm{C}$. Power is supplied by a rechargeable Li-Pol battery (6). Finally an iPod connector (7) is used as interface for data transfer, to recharge the battery and to program the microcontroller. Figure 6 shows an assembled mote.

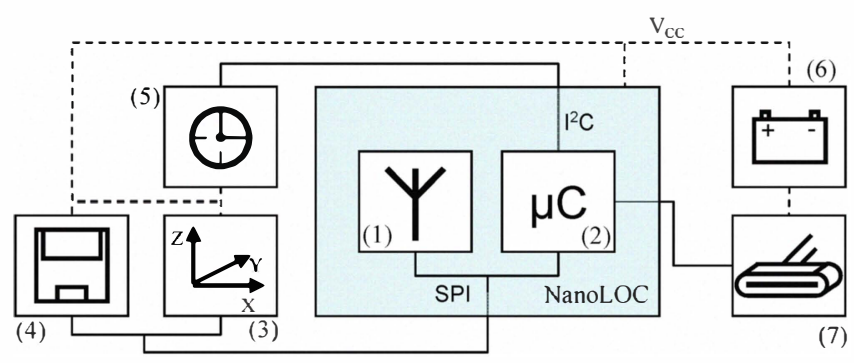

Figure 5. Hardware architecture of a mote.
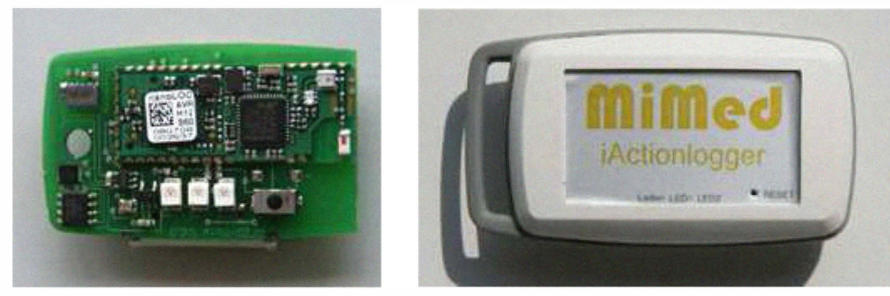

Figure 6. Assembled mote. 


\section{EXPERIMENT}

In order to prove functionality we defined four demands that the system should be capable to handle.

1) Setting of interaction radii: Once the interaction radius (transmitting range) of a passive mote is set, it will be recognized by an active motes that happens to be in a distance $<\mathrm{r}_{\mathrm{I}}$. If the distance between the motes is $>\mathrm{r}_{\mathrm{I}}$, the active mote cannot detect the passive mote.

2) Recognition of active and passive motes: Active motes are able to detect passive motes and other active motes, in which transmitting range they are.

3) Overlapping of interaction radii: If the interaction radii of several passive motes overlap, an active mote is able to recognize them separately.

4) Measuring of interaction time: The time of interaction measured by an active mote matches the actual interaction time.

\section{A. Experiment Setup}

The demands were transferred to the following experimental scenarios. They all imply that the test persons are carrying an active mote in their pocket.

1) Setting of interaction radii: A set interaction radius of a passive mote is marked by a line at the floor. Whenever a person crosses the line his/her mote detects a contact. Behind the line no contact is detected

2) Recognition of active and passive motes: The test persons are brought to contact situations with objects that are marked with active and passive motes. Each of them should be recognized.

3) Overlapping of interaction radii: An object $\mathrm{x}$ with $\mathrm{a}$ small interaction radius is arranged in the interaction radius of an object $y\left(r_{I x}<r_{I y}\right)$. Depending on the position of the test person, his active mote either recognizes both objects or only object y or none of them. If several subjects meet at a place that is marked with a passive mote. The active mote of each subject should recognize the passive mote as well as the other subjects' active motes.

4) Measuring of interaction time: During the experiment the test persons are watched by a minute taker who documents their interactions with marked objects. The duration of interactions recognized by their active motes match with the test minutes.

To carry out the experiment we marked objects and rooms at our department with active and passive motes and set their transmitting ranges using the initializing device. The objects/ rooms were: Our library, a table in the library, the kitchen and the coffee machine in the kitchen (see Figures 7 and 8). The settings in the library and kitchen represent the scenarios 3) and 2) (when more than one test participant is in the library). Besides these objects, a printer and two coffee mugs were endued with active motes (see Figures 9 and 10). The setting at the printer was used to explicitly scrutinize scenario 1). To ensure that the test persons are not influenced by seeing the motes when carrying out an activity, all motes were applied concealed. We provided three test persons that had no knowledge about the iActionlogger system with an active mote each and asked them to carry it in their pocket when performing different tasks. The motes were enveloped in yellow, red and blue coloured adhesive tape, which in consequence let to the later used term "yellow," "red" and "blue" when discussing the results of the obtained data (see Figure 11). Every test person was handed out a detailed list of tasks to perform. These included e.g. getting a cup of coffee, sitting together in the library discussing a text, going to the printer to pick up a paper and so on. The instructions only contained information about what to do and not how to do it. We wanted the test persons to carry out their actions as usually. The order of tasks differed for each test person. The experimental plan was designed in a way that each of the described scenarios was fulfilled by each test person at least twice. During the whole experiment each test person was watched by a minute taker who documented the carrying out of the tasks and the corresponding duration to check the accuracy of the recorded data after the experiment.

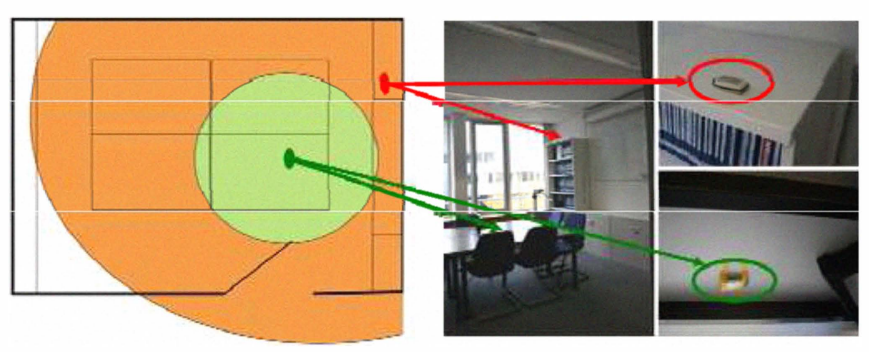

Figure 7. Motes marking the library and a table in the library.

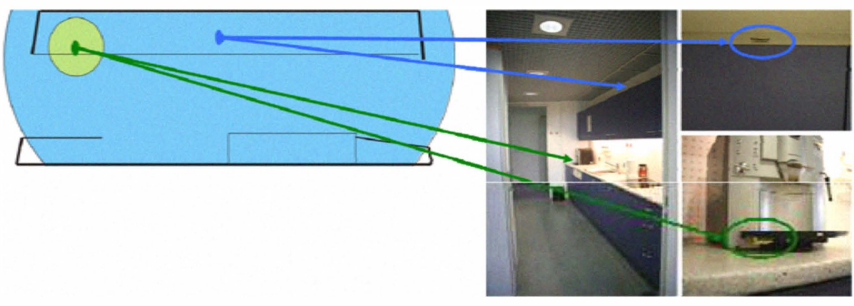

Figure 8. Motes marking the kitchen and the coffee machine.

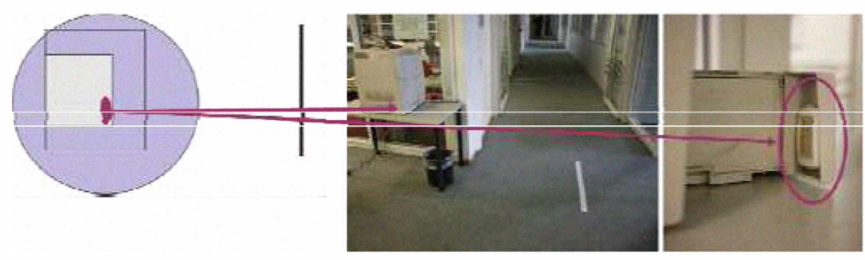

Figure 9. Mote marking the printer. 

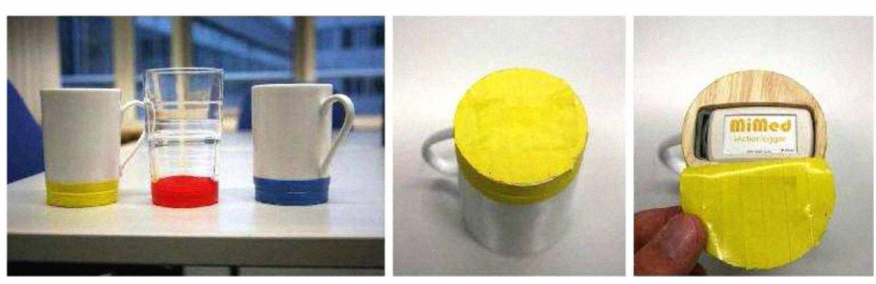

Figure 10. Coffee mug with applied mote

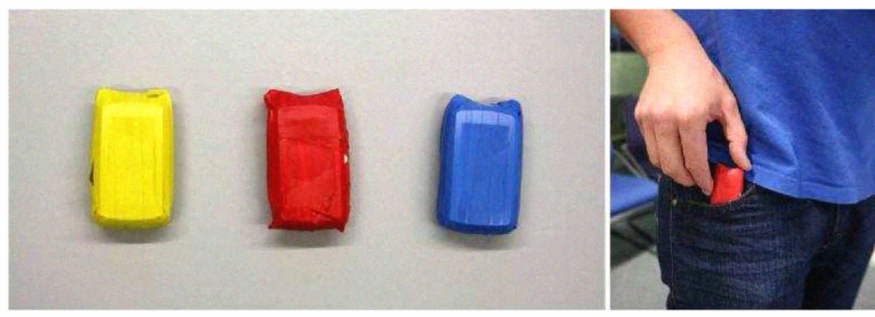

Figure 11. Active motes for test persons.

\section{B. Results}

After the experiment the saved data of each active mote (persons "yellow", "red" and "blue"; coffee mugs "yellow" and "blue" and "printer") was transmitted to a computer. Here the information was visualized as a Gantt chart for each active mote. In the graphical illustration the names of all motes are listed at the $\mathrm{y}$-coordinate whereas the $\mathrm{x}$-coordinate represents time. For every contact detected with a mote $\mathrm{x}$ the related line of mote $\mathrm{x}$ is marked according to the duration of contact. Based on the minutes of the experiment the actual duration of contact is also displayed in the graphs, which offers a very good opportunity to evaluate the accuracy of the system. In the following we will focus on four different situations that represent the four scenarios described above.

\section{1) Setting of Interaction Radii}

The setting of individual interaction radii is vital for the function principle of the whole system and, of course, was tested many times during the development in our lab. Without the ability to set the transmitting range the whole experiment would not lead to any usable results. Nevertheless we explicitly checked this requirement at the setting shown in Figure 9. The three test persons had to wait behind the line and step forward to the printer one after another to rupture a piece of paper and throw it in the garbage can. The results of recognized interaction are shown in figure 12 . The green boxes represent the time next to the printer according to the minutes whereas the orange boxes display the interaction duration recognized by the active motes. One can see that person "yellow" went to the printer first, followed by "red" and "blue". At the very beginning of the sequence "blue" and "red" both recognize the printer for a few seconds. That comes because when arriving at the printer station the test persons crossed the interaction radius of the printer before they got in line. "Red" perfectly recognized the printer as documented in the minutes. "Yellow" recognized the printer with a short delay compared to the minutes. "Blue" did not recognize the printer during the task at all. This can be explained with the fact that the test persons were not explicitly to interact with the printer but to use the garbage can next to the printer. There was no actual need to stand close to the printer to throw the paper away, which "blue" did not. This example shows how different even easy tasks can be fulfilled.

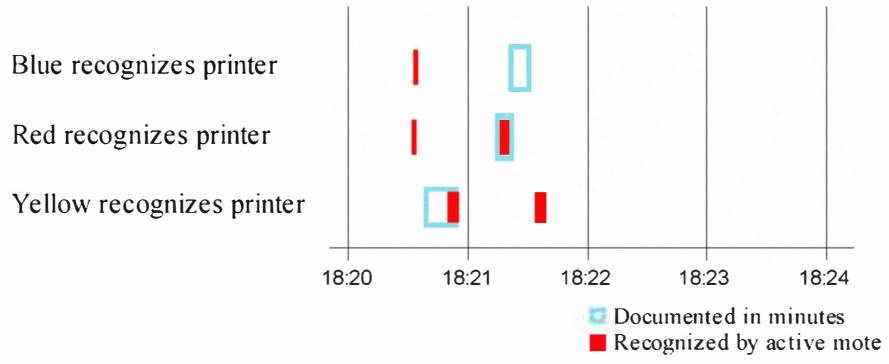

Figure 12. Contacts of test persons "blue", "red" and "yellow "with the mote hidden in the printer.

\section{2) Recognition of active and passive motes}

During the experiment on several occasions active and passive motes happened to be together within their transmitting ranges. Our demand on such situations is that the active motes recognize each other and the passive motes. Figure 13 shows an extract of the experiment. Test persons "red" and "blue" entered the library together and sat down at the table to read a text as next task. After about two minutes they both left the library to walk around the department together. When doing so the distance between them varied. This is why that period was not logged as a constant contact. Afterwards "blue" had to pick up a glass from the table and place it somewhere outside the library. Finally they both set down at the table again. Although the results from the minutes and the active motes do differ in this extract of the experiment (especially at the end), one can clearly see that during the first half the active motes recognized each other and the passive mote under the table and thus fulfill the second requirement.

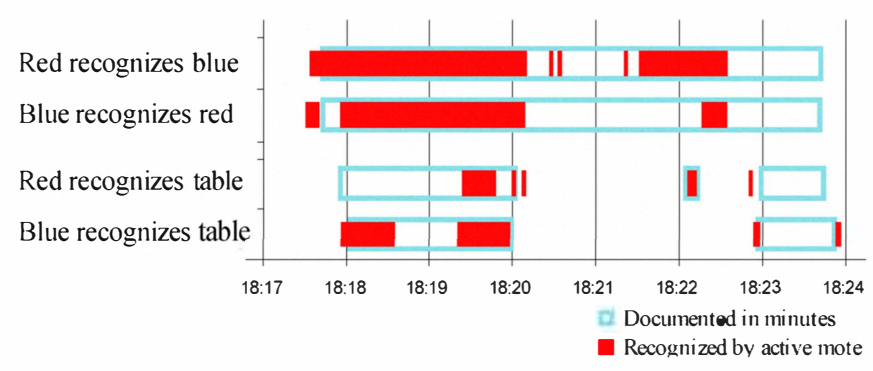

Figure 13. Contact times between test persons "blue", "red" and the table in the library.

\section{3) Overlapping interaction radii}

In respect of the goal to mark many objects for the detection of ADLs, the ability to recognize several motes with overlapping interaction radii is an important point. We checked this before the presented experiment several times while developing the system with a maximum of ten passive motes lying together at a table that all got recognized by an active mote. Due to our limited number of motes we only had two scenarios with two passive motes' ranges overlapping. These were as shown in Figure 7 and Figure 8, in the library and in the kitchen. The data logged by test person "yellow's" mote shows a good matching with the minutes when making coffee (see Figure 14). During the time in the library the table was not 
recognized when the person was sitting which could be caused by a covering of the radio signal. Despite this period one can clearly see that the mote "yellow" recognized all the passive motes as demanded.
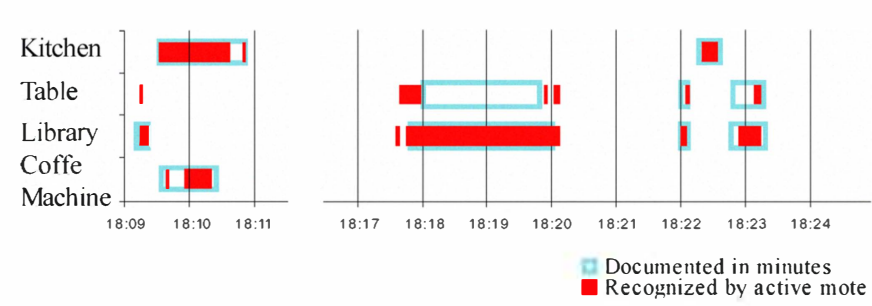

Figure 14. Contacts logged by the mote of test person "yellow"

\section{4) Measuring of interaction time}

In order to obtain a maximum of information about interactions our goal was to not only get Boolean results (contact $=$ true or false) but also quantitative time information (contact from ... until...). Our experiments showed that this criterion was achieved with varying success. As you can see in Figures 12-14, the minutes and the data of the motes do not always match exactly. When comparing them exact to the second, the worst matching quotient was $44 \%$ for the duration of the experiment (about 12 minutes) whereas the best lay above $90 \%$.

\section{Discussion}

The Results of the experiment prove that the concept of our system is working and worth to be developed further. The exact measuring of interaction time came out to be not as constant in quality as expected. In most instances this is surely based on the characteristic of the used $2,4 \mathrm{GHz}$ radio frequency. It is known that this frequency is absorbed by water very well. The interaction between the user and $2,4 \mathrm{GHz}$ radio waves can cause de-tuning effects and like water, the user's body absorbs energy of the transmitted radio signals. Metal surfaces reflect and also absorb the radio waves and thus distort the radiation pattern. This distortion impairs the quality of signal transmission. During the experiment we could see the influence of such effects. For example, empty coffee mugs were recognized much better than full ones. In the setting shown in figure 14 the test person's mote did not recognize the table when he was sitting next to it. From our minutes we know the person crossed his legs and sat in a very "easy" way. We assume that this led to a shielding of his mote and the result described above. In respect to the radio characteristics the initializing device turned out to be of a very high value. The transmitting range of a mote always depends on the surroundings and thus can not be set to $x$ metres in advance. The same transmitting power of a mote leads to different ranges in different environments.

\section{CONCLUSION}

In this article the system iActionlogger for the detection of interaction with objects was presented. The system is based on $2,4 \mathrm{GHz}$ radio modules to mark objects and to function as interaction logger. The transmitting ranges of the modules are set by an initializing device and thus individual interaction radii for different objects can be set by the user. The system offers an easy to install and not attracting attention possibility to mark objects in a home environment. The user does not need to carry his logger visible for anyone else and can wear it understated under his clothes e.g. in a pocked. Due to the small size of the logging device the user will not be affected in his usual carrying out of activities. Hence this is a promising approach for a high user acceptance.

In future work we will focus on analyzing functions for the obtained data. Our goal is to automatically recognize and document user ADLs with the iActionlogger as monitoring tool. At the moment further tests of the system that focus on the variation of different parameters are in preparation.

\section{ACHNNOWLEDGEMENT}

This work has received funding from the Bavarian Research Foundation (BFS) under contract number AZ-780-07. The views expressed here are those of the authors only. The BFS is not liable for any use that may be made of the information contained therein.

Within the research consortium of the Bavarian Research Foundation (BFS) „Fit4Age“ a team of scientists and engineers affiliated to 13 departments of the Bavarian universities Erlangen-Nürnberg, München, Regensburg and Würzburg works together with 25 industrial partners on the development of products and services for the aging society.

The scope of the research consortium is to develop technology based solutions which will help elderly people in their future living environment comprising home and workplace as well as in communication and transportation. Eventually not only elderly people but also all social groups should profit from these solutions.

\section{REFERENCES}

[1] J. Dobritz, A. Ette, K. Gärtner, E. Grünheid, R. Mai, F. Micheel, „Bevölkerung: Daten, Fakten, Trends zum demographischen Wandel in Deutschland,“ Wiesbaden: Bundesinstitut für Bevölkerungsforschung und Statistisches Bundesamt, 2008

[2] L. Fratiglioni, L. J. Launer, K. Andersen, M. M. B. Breteler, J. R. M. Copeland, J.-F. Dartigues, A. Lobo, J. Martinez-Lage, H. Soininen, A.Hofman, "Incidence of dementia and major subtypes in Europe : A collaborative study of population-based cohorts," in Neurology 54, pp. $10-15,2000$

[3] D. J. Cook and S.K. Das, "How smart are our environments? An updated look at the state of the art," Pervasive and Mobile Computing, Volume 3, Issue 2, pp. 53-73, 2007

[4] S. Katz, "Assessing Self-Maintenance: Activities of Daily Living, Mobility and Instrumental Activities of Daily Living," Journal of the American Geriatrics Society 31(12) pp. 721-726, 1983

[5] S. Suzuki, H. Asama, A. Uegaki, S. Kotosaka, F. Fujita, A. Matsumoto, H. Kaetsu, I. Endo, „An Infra-Red Sensory System with Local Communication for Cooperative Multiple Mobile Robots," IEEE, Proceedings of RSJ Conference 1995, pp. 220-225, August 1995

[6] Y. Arai, T. Fujii, H. Asama, Y. Kataoka, H. Kaetsu, A. Matsumoto, I.Endo, "Collision Avoidance among Multiple AutonomousMobile Robots using LOCISS (Locally Communicable Infrared Sensory System),“ IEEE, Proceedings of Robotics and Automation Conference 1996, pp 2091-2096, April 1996

[7] R. Want and A. Hopper, „Active Badges And Personal Interactive Computing Objects,“ IEEE, Consumer Electronics 1992, pp. 10-20 February 1992 
[8] R. Want, A. Hopper, V. Falcao, J. Gibbons, „The Active Badge Location System, " ACM Transactions on Information Systems (TOIS), Volume 10 , Issue 1, pp. 91-102, 1992

[9] G. LeBellego, N. Noury, G. Virone, M. Mousseau, J. Demongeot, „A Model for the Measurement of Patient Activity in a Hospital Suite," IEEE, Transactions on Information Technology in Biomedicine, Volume 10, Issue 1, pp. 92-99, 2006

[10] A. Harter, A. Hopper, P. Steggles, A. Ward, P. Webster, „The Anatomy of a Context-Aware Application," Selectet Papers from Mobicom, Wireless Networks, pp. 187-197, March 2002

[11] H. Balakrishnan, R. Baliga, D. Curtis, M. Goraczko, A. Miu, B. Priyantha, A. Smith, K. Steele, S. Teller, K. Wang, „Lessons from Developing and Deploying the Cricket Indoor Location System,“ Preprint, 2003
[12] Z. Uhrikova, C.Nuget, V. Hlavac, "The use of Computer Vision techniques to augment home based sensorised environments," IEEE, EMBS Conference, pp. 2550-2553, 2008

[13] K. Kim, K. Medioni, „Distributed Visual Processing for a Home Visual Sensor Network, "IEEE WACV, pp. 1-6, 2008

[14] M. Muira, S. Ito, R. Takatsuka, S. Kunifuij, „Aware Group Home Enhanced by RFID Technology," Knowledge-Based Intelligent Infromation and Engineering Systems, Berlin/Heidelberg: SpringerVerlag, pp. 847-854, 2008

[15] K.P. Fishkin, M. Philipose, A.Rea, „Hands-On RFID: Wireless Wearables for Detecting Use of Objects, " IEEE Wearable Computers, pp. 38-41, 2005

[16] C. Lustig, H. Novatchkov, L. E. Dunne, M. McHugh, L. Coyle, „Using Colocation to Support Human Memory", MeMos 2007: Supporting Human Memory with interactive Systems, pp.41-44, 2007 